() А.А. Мосикян ${ }^{1}$, А.Ю. Бабенко ${ }^{1 *}$, Ю.А. Севастьянова², Р.В. Драй², Е.В. Шляхто1

'Национальный медицинский исследовательский центр им. В.А. Алмазова, Санкт-Петербург

${ }^{2} Г К$ «Герофарм», Санкт-Петербург

ОБОСНОВАНИЕ. Индивидуализированный подход к назначению терапии в настоящее время из теории становится частью рутинной клинической практики. Уже накоплено немало данных по предикторам гипогликемической эффективности препаратов класса ингибиторов дипептидилпептидазы-4 (иДПП-4), представленных на международном рынке, однако подобных работ для нового лекарственного препарата - эвоглиптина - до сих пор не проводилось.

ЦЕЛЬ. Определить клинико-лабораторные предикторы гипогликемической эффективности эвоглиптина.

МЕтоды. Ретроспективное исследование базы данных ранее проведенного рандомизированного клинического испытания по сравнению эффективности и безопасности эвоглиптина и ситаглиптина, построение однофакторных и многофакторной модели линейной регрессии для показателя «снижение гликированного гемоглобина (HbA $\left.{ }_{1 c}\right)$ через 24 недели терапии».

РЕЗУЛЬТАТЫ. В российской субпопуляции снижение $\mathrm{HbA}_{1 с}$ через 24 нед терапии отрицательно коррелировало со значением соотношения концентраций триглицеридов и липопротеидов высокой плотности (ЛПВП) - метаболическим

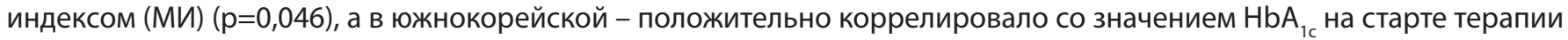
( $p<0,0001)$. Для повышения статистической мощности данные российской и корейской субпопуляций были объединены. При объединении данных была получена положительная корреляция с исходным $\mathrm{HbA}_{1 c}(\mathrm{p}<0,0001)$ и со значением десятичного логарифма индекса НОМА-B ( $p=0,0042)$, а также - отрицательная со значением десятичного логарифма МИ ( $p=0,0057)$, концентрацией фосфора в венозной крови $(p=0,014)$ и приемом статинов $(0,044)$. Не было получено корреляции между снижением $\mathrm{HbA}_{1 с}$ через 24 нед и индексом массы тела, длительностью сахарного диабета (СД) и концентрацией С-пептида в плазме крови. У пациентов, достигших целевого значения $\mathrm{HbA}_{1 c}<7,0 \%$ через

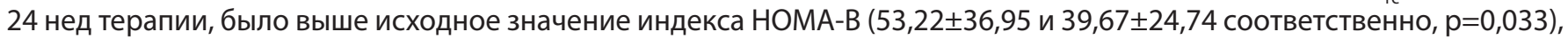

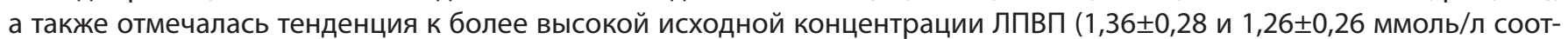
ветственно, $\mathrm{p}=0,076)$ и к более низкому МИ $(0,87 \pm 0,70$ и 1,48 $\pm 0,95$ соответственно, $\mathrm{p}=0,079)$.

ЗАКЛЮЧЕНИЕ. Пациент, который получит максимальную гликемическую выгоду от применения эвоглиптина, это пациент с более высоким индексом НОМА-В, с меньшим МИ и с низко-нормальной концентрацией фосфора в плазме крови. МИ при этом является предиктором, специфическим для российской популяции. Полученные данные поднимают вопрос о наличии различий в предикторах ответа на терапию отдельными представителями класса иДПП-4, что требует тщательного анализа предикторов для каждого представителя этого класса.

КЛЮЧЕВЫЕ СЛОВА: сахарный диабет; персонализированная медицина; иДПП-4; предикторы ответа; эвоглиптин

\title{
EFFECTIVENESS PREDICTION OF EVOGLIPTIN TREATMENT IN TYPE 2 DIABETES MELLITUS IN RUSSIAN-KOREAN POPULATION
}

\author{
(c) Anna A. Mosikian', Alina Y. Babenko1* , Yulia A. Sevastyanova², Roman V. Drai², Evgenij V. Shlyakhto'
}

${ }^{1}$ National Almazov North-West Medical Research Centre, Saint-Petersburg, Russia

${ }^{2}$ Geropharm, Pharmaceutical Company, Saint-Petersburg, Russia

BACKGROUND: Individualized treatment has already become a part of a routine clinical care. Many data on the effectiveness prediction of commercially available DPP-4 inhibitors had been published, but not on the effectiveness prediction of evogliptin.

AIM: To reveal the clinical characteristics and metabolic predictors of better hypoglycemic response to evogliptin. Matherials and methods: We have conducted a retrospective study, based on the data of a randomized clinical trial comparing effectiveness and safety of evogliptin and sitagliptin in Russian and Korean subpopulations. We provide univariate linear regression models for separate subpopulations and a multivariate stepwise regression model for the combined subpopulation. $\mathrm{HbA}_{1 \mathrm{c}}$ change after 24 weeks of evogliptin treatment was a primary endpoint and a dependent variable in the analysis.

RESULTS: The decrease of $\mathrm{HbA}_{1 c}$ after 24 weeks of treatment with evogliptin in Russian subpopulation negatively correlates with triglycerides/HDL level $(p=0,046)$. In South Korean subpopulation it correlates positively with $\mathrm{HbA}_{1 c}$ level at baseline 
$(p<0,0001)$. In order to increase the statistical power of the analysis the data of both populations were combined. According to the combined data, the decrease of $\mathrm{HbA}_{1 c}$ after 24 weeks of treatment with evogliptin positively correlates with $\mathrm{HbA}_{1 c}$ level at baseline $(p<0.0001)$ and $\log (\mathrm{HOMA}-\mathrm{B})(p=0.0042)$, and it negatively correlates with log(triglycerides/HDL) $(p=0.0057)$, blood phosphorous concentration ( $p=0.014)$ and statin treatment $(p=0.044)$. No correlation of $\mathrm{HbA}_{1 c}$ change at week 24 was observed with body mass index, diabetes duration and blood C-peptide concentration. Patients able to achieve $\mathrm{HbA}_{1<}<7,0$ $\%$ had higher HOMA-B $(53.22 \pm 36.95$ и $39.67 \pm 24.74$, respectively, $\mathrm{p}=0.033)$ and were tend to have higher HDL concentration $(1.36 \pm 0.28$ и $1.26 \pm 0.26 \mathrm{mmol} / \mathrm{l}$, respectively, $\mathrm{p}=0.076)$ and lower triglycerides to HDL ratio $(0.87 \pm 0.70$ и $1.48 \pm 0.95$, respectively, $\mathrm{p}=0.079$ ).

CONCLUSION: A patient, who benefits more when treated with evogliptin, has higher HOMA-B, lower triglycerides to HDL ratio and phosphorous concentration in the 1-2 quartiles of the normal range. Triglycerides to HDL ratio is, probably, a specific effectiveness predictor for Russian, but not for Korean subpopulation. These data prove the difference in effectiveness prediction for different drugs of DPP-4 inhibitors group and reveal the need of further investigation.

KEY WORDS: diabetes mellitus; personalized medicine; DPP-4 inhibitors; efficacy prediction; evogliptin

В последние годы концепция персонализированной медицины (personalized medicine) стала важной частью развития современной фармакотерапии [1]. Ключевая концепция такого подхода, «лечение верным препаратом в верной дозе верного пациента» ("therapy with the right drug at the right dose in the right patient"), была определена в 2000 г. [2] и по настоящее время не изменилась. Точная медицина (precision medicine) является одновременно и более актуальным названием для персонализированной медицины, и одним из компонентов персонализированной медицины и концентрируется прежде всего на подборе лекарственного средства с учетом индивидуальных характеристик пациента, а не на разработке уникальных лекарственных средств для малых групп потребителей [3]. Точная медицина становится особенно актуальной при необходимости назначения лекарственных препаратов на длительный срок при хронических заболеваниях.

Эвоглиптин - гипогликемическое лекарственное средство класса ингибиторов дипептидилпептидазы-4 (иДПП-4), назначаемое пациентам с сахарным диабетом 2 типа (СД2) [4]. Для данного класса гипогликемических лекарственных препаратов к настоящему моменту накоплено немало информации о факторах, ассоциированных с индивидуальной вариабельностью гипогликемического ответа. Снижение гликированного гемоглобина $\left(\mathrm{HbA}_{1 c}\right)$ было тем более выражено, чем выше был $\mathrm{HbA}_{1 с}$ на старте терапии [5, 6], но в то же время иДПП-4 обладают умеренным гипогликемическим эффектом [5], в связи с чем ответ на терапию по параметру $\mathrm{HbA}_{1 c}<7,0 \%$ ожидается у пациентов с умеренным повышением данного показателя: вероятность достижения целевого значения $\mathrm{HbA}_{1 c}$ понижается на $36 \%$ с увеличением $\mathrm{HbA}_{1 c}$ на $1 \%$ [7]. Эта корреляция подтверждается и в более раннем мета-анализе [8]. Эффективность иДПП-4 также отрицательно коррелирует с длительностью течения СД2 [7], индексом массы тела (ИМТ) и анамнезом ишемической болезни сердца [6]. Кроме того, назначение иДПП-4 в комбинации с метформином повышает эффективность терапии в 2,6 раза по сравнению с монотерапией иДПП-4, тогда как совместное назначение иДПП-4 и комбинации метформина и препарата сульфонилмочевины только на 42\% эффективнее монотерапии иДПП-4 с точки зрения вероятности достижения целевых значений $\mathrm{HbA}_{1 c}$ (<7,0\%) [7]. При добавлении иДПП-4 к монотерапии препаратами сульфонилмочевины вероятность достижения оптимального контроля гликемии $(<7,0 \%)$ повышалась менее чем на 35\%, и это повышение не было статистически значимым ( $p=0,07)$. Добавление иДПП-4 к монотерапии препаратами сульфонилмочевины, по-видимому, вовсе не является оправданным, так как дополнительное снижение $\mathrm{HbA}_{1 c}$ не превышало 0,25\% в группе пациентов с исходным $\mathrm{HbA}_{1 с}$ в диапазоне 7,5-9,0\% $(\mathrm{p}=0,10)$ и было равным в среднем 0,88\%, если исходный $\mathrm{HbA}_{1 с}$ превышал $9,0 \%(p=0,08)[7]$.

Еще одним фактором, ассоциированным с большей эффективностью иДПП-4, является азиатский этнос пациента [9], однако авторы мета-анализа, по результатам которого выделен данный фактор, не исключают роль конфаундинга: ИМТ пациентов азиатской этнической группы был меньше, чем у европеоидной группы, при этом ИМТ коррелирует с чувствительностью к инсулину [10], которая различается у этнических групп [11]. Тем не менее полученные результаты остаются статистически значимыми после поправки на ИМТ [9]. Кроме того, в малазийской популяции было показано, что пациенты с содержанием триглицеридов $<1,7$ ммоль/л $(p=0,041)$, диастолическим артериальным давлением <90 мм рт. ст. $(\mathrm{p}=0,046)$ и KCNJ11 rs2285676 (генотип CC) $(\mathrm{p}=0,042)$ чаще достигают $\mathrm{HbA}_{1 c}<7,0 \%$ [12].

При этом следует отметить, что KCNJ11 является геном, наиболее часто обсуждаемым в качестве предиктора ответа на терапию препаратами сульфонилмочевины, а не иДПП-4 [13]. Признанным генетическим предиктором ответа на иДПП-4, в свою очередь, является CTRB1/2 rs7202877 (генотип ТT) - у носителей данного генотипа было зарегистрировано снижение $\mathrm{HbA}_{1 c^{\prime}}$ на 0,5\% большее, чем у носителей генотипа CTRB1/2*TG, при этом 84\% пациентов исследуемой субпопуляции получали ситаглиптин [14]. Среди других генов, варианты которых ассоциированы с риском развития СД2, особенно следует выделить TCF7L2 rs7903146. Носители TCF7L2*TT имели меньшее снижение $\mathrm{HbA}_{1 c}$ в ответ на терапию линаглиптином по сравнению с носителями TCF7L2*CC $(0,57 \%$ и 0,82\% соответственно, $p=0,018)$, а эффективность линаглиптина у носителей TCF7L2*CT не отличалась от таковой у носителей TCF7L2*CC [15].

К настоящему времени также накоплены данные о клинико-лабораторных факторах, влияющих на эффективность отдельных представителей данной группы. Была получена информация о прямой корреляции снижения $\mathrm{HbA}_{1 c}$ и концентрации С-пептида в плазме крови натощак при приеме ситаглиптина и вилдаглиптина $[16,17]$. Однако эффективность иДПП-4 уменьшается 
Таблица 1. Ковариаты, включенные в первичный анализ

Демографические параметры

Пол, возраст

Антропометрические параметры

Индекс массы тела

Инструментальные показатели

Систолическое и диастолическое артериальное давление

Расчетные индексы

HOMA-IR, HOMA-B, QUICKI, ТГ/ЛПВП

Прочие лекарственные препараты

иАПФ, БРА, бета-блокаторы, статины

\section{Лабораторные показатели}

Гликемический контроль $\left(\mathrm{HbA}_{1 c^{\prime}}\right.$ глюкоза плазмы натощак, концентрация С-пептида и инсулина)

Показатели липидограммы (общий холестерин, лПНП, ЛПВП, триглицериды)

Ионный состав крови (натрий, калий, кальций, фосфор, хлорид анион) прочие показатели (креатинин, альбумин в сыворотке крови, концентрация АЛТ)

Анамнестические данные

АГ, ИБС, ХСН, длительность СД по мере увеличения инсулинорезистентности. Отсутствие метаболического синдрома было ассоциировано с более выраженным снижением $\mathrm{HbA}_{1 c}$ при приеме линаглиптина [18]. В то же время было показано, что при смене препарата сульфонилмочевины на ситаглиптин или вилдаглиптин более высокий индекс инсулинорезистентности (HOMA-IR) ассоциирован с большей эффективностью последних относительно исходной терапии [17]. Вероятным объяснением этого факта является более высокая экспрессия ДПП-4 как в подкожной, так и в висцеральной жировой ткани [19] и, соответственно, большее количество субстрата для действия иДПП-4.

Для ситаглиптина в отдельности показана прямая корреляция между эффективностью лекарственного препарата и постпрандиальной глюкозой плазмы крови [20]. Между тем есть предикторы, имеющие разнонаправленное значение при терапии отдельными представителями данной группы. Так, обнаружена отрицательная корреляция между эффективностью ситаглиптина и возрастом пациентов [20, 21], тогда как алоглиптин продемонстрировал более высокую эффективность у пациентов старше 65 лет [22]. В то же время было показано, что иДПП-4 как класс препаратов имеют большую эффективность, чем метформин, у пожилых пациентов в качестве первой линии терапии [8], однако в более поздних исследованиях не было выявлено статистически значимых корреляций между эффективностью класса иДПП-4 в целом и возрастом пациента [5, 7].

Таким образом, накопленные данные позволяют выделить потенциальные факторы, ассоциированные с эффективностью эвоглиптина как представителя класса иДПП-4.

\section{ЦЕЛЬ}

Настоящее исследование имело целью определить уникальные факторы, ассоциированные с выраженностью гипогликемического эффекта эвоглиптина.

\section{МЕТОДЫ}

\section{Дизайн исследования}

Ретроспективное исследование на основании предоставленной базы данных ранее проведенного двойного слепого рандомизированного клинического испытания по сравнению эффективности и безопасности эвоглипти- на 5 мг и ситаглиптина 100 мг.

\section{Критерии соответствия}

В анализ были включены данные 140 пациентов с СД2 на монотерапии метформином, принимавших эвоглиптин в дозе 5 мг в рамках двойного слепого контролируемого рандомизированного клинического испытания по сравнению эффективности и безопасности эвоглиптина 5 мг и ситаглиптина 100 мг. Пациенты принимали препарат ежедневно в течение 24 нед. Эффективность гипогликемической терапии оценивалась по изменению $\mathrm{HbA}_{1 c}$ через 24 нед относительно исходного уровня (непосредственно перед началом терапии).

\section{Условия проведения}

Пациенты, данные которых были включены в настоящий анализ, были рекрутированы на базе 33 клинических центров, 6 из которых расположены на территории Российской Федерации, а 27 центров - на территории Южной Кореи.

\section{Продолжительность исследования}

Клиническое исследование, данные которого были использованы для настоящего анализа, состояло из периода скрининга (2 нед) и периода лечения (24 нед).

\section{Основной исход исследования}

Поскольку настоящее исследование является ретроспективным и имеет целью определить факторы, ассоциированные с лучшим гипогликемическим эффектом эвоглиптина, понятие первичной конечной точки в классическом понимании в данном случае неприменимо. При нижеописанном подходе к анализу данных исходами исследования являются все факторы, имеющие статистическую значимость.

\section{Ковариаты, включенные в анализ}

В первичный анализ были включены ковариаты, которые коррелировали с эффективностью других иДПП-4 по данным обзора литературы, а также не вошедшие в этот перечень ковариаты, влияние которых предполагалось в связи с их клинической значимостью. Дополнительно были рассчитаны значения индексов инсулинорезистентности HOMA-IR и функции бета-клеток HOMA-B, количественный индекс контроля чувствительности 
к инсулину QUICKI (quantitative insulin-sensitivity check index) $[23,24]$ и соотношение содержания триглицеридов к липопротеидам высокой плотности (ЛПВП) - метаболический индекс (МИ) $[25,26]$. Список включенных в первичный анализ ковариат приведен в табл. 1. Все значения были получены непосредственно перед началом терапии.

\section{Методы регистрации исходов}

Регистрация всех демографических, антропометрических, лабораторных, инструментальных и анамнестических ковариат производилась в соответствии с протоколом исследования ЭВОКОМБИ при его проведении. В настоящем исследовании новые исходы не регистрировались.

Индекс HOMA-IR рассчитывался по формуле: HOMA-IR = ((глюкоза натощак (ммоль/л)) * (инсулин натощак (мкЕд/мл))) / 22,5.

Индекс НОМА-В рассчитывался по формуле: НОМА-B $=(20$ * инсулин натощак (мкЕд/мл))/ (глюкоза натощак (ммоль/л) - 3,5).

Индекс QUICKI рассчитывался по формуле: QUICKI = 1 / (log (инсулин натощак (мкЕд/мл)) + $\log$ (глюкоза натощак (ммоль/л) * 18).

\section{Этическая экспертиза}

Все основные документы исследования ЭВОКОМБИ были представлены в независимые этические комитеты исследовательских центров, согласно установленным процедурам таких комитетов. Условием для проведения клинического исследования являлось Разрешение №129 МЗ РФ от 20.02.2016 г. и одобрение исследования Советом по этике (Заключение заседания Совета по этике №118 от 02.02.2016 г.). В Корее разрешение Министерства по безопасности пищевых продуктов и медикаментов (Ministry of Food and Drug Safety) на проведение клинического исследования было получено 11.04.2013 г. (Разрешение №11953). Разрешение на предоставление данных ГК «Герофарм» для проведения ретроспективного анализа было получено.

\section{Статистический анализ}

Базовые характеристики включенных пациентов представлены в виде среднее \pm стандартное отклонение - для непрерывных переменных или количество пациентов (\%) - для категориальных данных.

Первичная конечная точка оценки эффективности эвоглиптина - изменение $\mathrm{HbA}_{1 с}$ через 24 нед терапии. Для определения факторов, коррелирующих с эффективностью терапии эвоглиптином через 24 нед в российской и корейской субпопуляциях по отдельности, была использована однофакторная модель линейной регрессии. Анализ объединенной базы данных производился с использованием многофакторной модели линейной регрессии (stepwise regression model). Для оценки вероятности влияния конфаундеров на коэффициенты полученной многофакторной модели дополнительно была использована однофакторная модель линейной регрессии с определением коэффициентов B и r для факторов, включенных в многофакторную модель.

Вторичная конечная точка оценки эффективности эвоглиптина - достижение пациентом $\mathrm{HbA}_{1 c} \leq 7,0 \%$ че- рез 24 нед терапии. Сравнение групп (достигли $\leq 7,0 \%$ и не достигли $\leq 7,0 \%$ ) производилось посредством критерия Манна-Уитни - для непрерывных переменных и теста хи-квадрат с поправкой Йейтса на непрерывность для бинарных переменных.

Значение $\mathrm{p}<0,05$ соответствовало статистической значимости. Недостающие данные не замещались, пациенты с отсутствующим значением переменной, используемой в многофакторной линейной модели, исключались из анализа. Статистический анализ был проведен с использованием программного пакета R x64 version 3.2.3 for Windows.

\section{РЕЗУЛЬТАТЫ}

\section{Объекты исследования}

В ретроспективный анализ были включены данные 132 пациентов, получавших эвоглиптин в рамках исследования ЭВОКОМБИ, у которых были зарегистрированы все конечные точки по показателю «уровень гликированного гемоглобина» через 24 нед от момента рандомизации.

\section{Характеристики пациентов в начале терапии}

Исходные характеристики включенных в анализ пациентов представлены в табл. 2. Выборка была сбалансирована по полу (46\% мужчин). 21\% пациентов получали препарат в России и 79\% - в Южной Корее. Средний возраст составил 57,2 года, средняя длительность диабета - 8,3 лет, средний $\mathrm{HbA}_{1 c}-7,45 \%$, С-пептида - 2,31 нг/мл и ИМТ - 26,5 кг/M². Все пациенты на старте исследования получали метформин, 45,7\% - статины, и 44,3\% - средства, влияющие на ренин-ангиотензин-альдостероновую систему (РААС). Пациенты, включенные в исследование в России, по сравнению с пациентами из Южной Кореи, имели более высокий гликемии натощак на старте терапии ( $p=0,039)$, более высокую концентрацию C-пептида в крови $(p=0,002)$, больший ИМТ $(p<0,0001)$, а также более выраженную дислипидемию ( $p=0,0003$ для концентрации общего холестерина; $p<0,0001$ для концентрации ЛПНП), однако они не различались по $\mathrm{HbA}_{1 c}$ и индексам инсулинорезистентности на старте терапии. Пациенты в российской субпопуляции имели более высокую скорость клубочковой фильтрации $(\mathrm{p}=0,013)$. Артериальная гипертензия чаще была диагностирована у российских пациентов ( $p=0,016)$, которые чаще, чем корейские пациенты, получали терапию ингибиторами ангиотензинпревращающего фермента (АПФ) ( $<<0,0001)$, при этом контроль артериального давления был удовлетворительным и сопоставимым ( $p=0,316$ для систолического АД и $\mathrm{p}=0,568$ для диастолического АД).

\section{Однофакторные модели линейной регрессии}

Однофакторные модели линейной регрессии для российской субпопуляции были построены с использованием данных 27 человек, для которых была определена первичная конечная точка. Среднее снижение $\mathrm{HbA}_{1 c}$ составило 0,55\% (95\% ДИ: 0,31-0,80\%). Выраженность снижения $\mathrm{HbA}_{1 c}$ через 24 нед отрицательно коррелировала со значением МИ ( $B=-0,320 \pm 0,153 ; p=0,046)$, при этом сила связи была слабой $(r=0,38)$. Также имелась тенденция к отрицательной корреляции между снижением $\mathrm{HbA}_{1 с}$ и концентрацией в плазме крови триглицеридов 
Таблица 2. Характеристики пациентов в начале терапии (среднее \pm стандартное отклонение)

\begin{tabular}{|c|c|c|c|c|}
\hline \multirow{2}{*}{ Параметр } & \multicolumn{4}{|c|}{ Значение } \\
\hline & Вся группа & Россия & Ю. Корея & p* \\
\hline Количество человек & 132 & 27 & 105 & - \\
\hline Пол (м:ж), \% & $45,7: 54,3$ & $46,4: 53,6$ & $44,8: 55,2$ & 1,00 \\
\hline Возраст, лет & $57,24 \pm 9,51$ & $56,14 \pm 9,79$ & $57,52 \pm 9,46$ & 0,620 \\
\hline Длительность СД, лет & $8,27 \pm 5,44$ & $7,28 \pm 5,12$ & $8,50 \pm 5,50$ & 0,295 \\
\hline Этническая принадлежность (рус:кор), \% & $20,7: 79,3$ & - & - & - \\
\hline $\mathrm{HbA}_{1 c^{\prime}} \%$ & $7,45 \pm 0,75$ & $7,55 \pm 0,88$ & $7,42 \pm 0,71$ & 0,696 \\
\hline Гликемия натощак, ммоль/л & $7,56 \pm 1,70$ & $8,28 \pm 2,42$ & $7,37 \pm 1,41$ & $0,039+$ \\
\hline С-пептид, нг/мл & $2,31 \pm 1,19$ & $2,99 \pm 1,70$ & $2,14 \pm 0,95$ & $0,002+$ \\
\hline Инсулин, мкЕд/мл & $8,80 \pm 6,52$ & $9,25 \pm 10,82$ & $8,68 \pm 4,86$ & 0,290 \\
\hline HOMA-B & $47,98 \pm 33,03$ & $48,51 \pm 47,90$ & $47,84 \pm 28,18$ & 0,192 \\
\hline HOMA-IR & $2,99 \pm 2,57$ & $3,34 \pm 4,27$ & $2,90 \pm 1,90$ & 0,500 \\
\hline QUICKI & $0,15 \pm 0,01$ & $0,15 \pm 0,02$ & $0,15 \pm 0,01$ & 0,499 \\
\hline ИМТ, кг/м² & $26,51 \pm 4,38$ & $30,48 \pm 4,81$ & $25,47 \pm 3,62$ & $<0,0001 \dagger$ \\
\hline Холестерин общий, ммоль/л & $4,52 \pm 1,01$ & $5,17 \pm 1,00$ & $4,35 \pm 0,95$ & $0,0003+$ \\
\hline ЛПВП, ммоль/л & $1,33 \pm 0,28$ & $1,30 \pm 0,32$ & $1,33 \pm 0,26$ & 0,544 \\
\hline ЛПНП, ммоль/л & $2,76 \pm 1,00$ & $3,73 \pm 0,81$ & $2,50 \pm 0,89$ & $<0,0001 \dagger$ \\
\hline Триглицериды (ТГ), ммоль/л & $1,60 \pm 0,80$ & $2,01 \pm 1,14$ & $1,49 \pm 0,64$ & 0,057 \\
\hline Метаболический индекс (ТГ/ЛПВП) & $1,30 \pm 0,79$ & $1,72 \pm 1,17$ & $1,19 \pm 0,79$ & 0,079 \\
\hline Систолическое АД, мм рт. ст. & $125,77 \pm 11,38$ & $127,86 \pm 10,19$ & $125,23 \pm 11,66$ & 0,316 \\
\hline Диастолическое АД, мм рт. ст. & $78,37 \pm 8,16$ & $77,32 \pm 6,41$ & $78,64 \pm 8,56$ & 0,568 \\
\hline Креатинин сыворотки крови, мкмоль/л & $74,81 \pm 17,60$ & $68,97 \pm 13,60$ & $76,34 \pm 18,25$ & $0,043+$ \\
\hline СКФ, мл/мин/1,73 м² (MDRD) & $84,44 \pm 21,47$ & $92,19 \pm 27,10$ & $82,49 \pm 21,29$ & $0,013+$ \\
\hline Альбумин в сыворотке крови, г/л & $45,79 \pm 2,41$ & $46,23 \pm 2,07$ & $45,67 \pm 2,52$ & 0,344 \\
\hline АЛТ, Ед/л & $26,60 \pm 14,44$ & $23,90 \pm 9,55$ & $27,31 \pm 15,42$ & 0,611 \\
\hline Натрий сыворотки крови, ммоль/л & $140,65 \pm 2,12$ & $141,03 \pm 1,74$ & $140,55 \pm 2,20$ & 0,306 \\
\hline Калий сыворотки крови, ммоль/л & $4,48 \pm 0,44$ & $4,62 \pm 0,44$ & $4,44 \pm 0,43$ & $0,046+$ \\
\hline Фосфор сыворотки крови, ммоль/л & $1,14 \pm 0,17$ & $1,07 \pm 0,16$ & $1,15 \pm 0,17$ & $0,031+$ \\
\hline Сердечно-сосудистые заболевания, \% & & & & \\
\hline Артериальная гипертензия & 65,0 & 85,8 & 59,0 & $0,016 \dagger$ \\
\hline ИБС & 7,9 & 17,9 & 5,7 & 0,092 \\
\hline $\mathrm{XCH}$ & 2,1 & 7,1 & 1,0 & 0,214 \\
\hline Статины, \% & 45,7 & 46,4 & 44,8 & 1,00 \\
\hline Средства, влияющие на РААС, \% & 44,3 & 75,0 & 35,2 & $0,0004+$ \\
\hline иАПФ & 11,4 & 46,4 & 1,9 & $<0,0001 \dagger$ \\
\hline Сартаны & 32,9 & 28,6 & 33,3 & 0,802 \\
\hline
\end{tabular}

Примечание: * сравнение групп «Россия» и «Южная Корея» проводилось с использованием критерия Манна-Уитни; †р<0,05.

( $B=-0,319 \pm 0,157 ; p=0,052)$, альбумина $(B=-0,155 \pm 0,087 ;$ $p=0,087)$ и фосфора ( $B=-2,039 \pm 1,148 ; p=0,087)$, однако количество наблюдений не позволило достичь статистической значимости. Изменения $\mathrm{HbA}_{1 с}$ в данном случае не коррелировали с $\mathrm{HbA}_{1 c}$ на старте терапии, что также подтверждает недостаточность выборки, поскольку данная корреляция отмечается для всех лекарственных препаратов с гипогликемическим эффектом.

Аналогичный анализ был проведен для южнокорейской субпопуляции (105 пациентов). В данном случае была получена положительная корреляция изменения $\mathrm{HbA}_{1 с}$ через 24 нед с исходным $\mathrm{HbA}_{1 с}(\mathrm{~B}=0,530 \pm 0,071$; $\mathrm{p}<0,0001$; средняя сила связи: $r=0,59)$ и с уровнем гликемии натощак на старте терапии $(B=0,116 \pm 0,041 ; \mathrm{p}=0,0054$; однако сила связи в данном случае была очень слабой: $r=0,29)$, при этом велика роль внутренней корреляции между $\mathrm{HbA}_{1 c}$ и гликемией натощак $(r=0,68)$. Других корреляций при анализе корейской субпопуляции получено не было. Среднее снижение $\mathrm{HbA}_{1 с}$ через 24 нед составило 0,60\% (95\% ДИ: 0,48-0,72\%). 
Таблица 3. Факторы, ассоциированные с эффективностью эвоглиптина через 24 недели лечения

\begin{tabular}{|c|c|c|c|c|c|c|}
\hline & \multicolumn{3}{|c|}{ Многофакторная модель } & \multicolumn{3}{|c|}{ Однофакторная модель } \\
\hline & $\begin{array}{c}\text { Коэффициент } \\
\text { линейной } \\
\text { регрессии (В) }\end{array}$ & $\begin{array}{c}\text { Стандартная } \\
\text { ошибка B }\end{array}$ & $\mathbf{P}$ & $\begin{array}{c}\text { Коэффициент } \\
\text { линейной } \\
\text { регрессии (В) }\end{array}$ & $\begin{array}{c}\text { Стандартная } \\
\text { ошибка B }\end{array}$ & $\mathbf{P}$ \\
\hline Интерсепт & $-3,158$ & 0,817 & - & - & - & - \\
\hline $\mathrm{HbA}_{1 c^{\prime}} \%$ & 0,515 & 0,078 & $<0,0001$ & 0,434 & 0,076 & $<0,0001$ \\
\hline $\log (\mathrm{HOMA}-\mathrm{B})$ & 0,252 & 0,086 & 0,0042 & 0,001 & 0,092 & 0,99 \\
\hline Log(ТГ/ЛПВП) & $-0,275$ & 0,098 & 0,0057 & $-0,134$ & 0,109 & 0,22 \\
\hline Фосфор & $-0,773$ & 0,309 & 0,014 & $-0,745$ & 0,348 & 0,034 \\
\hline Статины (реф - нет) & $-0,223$ & 0,110 & 0,044 & $-0,098$ & 0,123 & 0,43 \\
\hline
\end{tabular}

Таблица 4. Различия групп пациентов, достигших и не достигших целевого значения $\mathrm{HbA}_{1 c} \leq 7,0 \%$

\begin{tabular}{lccc}
\hline \multicolumn{1}{c}{ Параметр сравнения, на старте терапии } & $\mathbf{H b A}_{\mathbf{1 c}} \leq \mathbf{7 , 0 \%}$ & $\mathbf{H b A}_{\mathbf{1 c}}>\mathbf{7 , 0 \%}$ & $\mathbf{p}$ \\
\hline $\mathrm{HbA}_{1 c^{\prime}} \%$ & $7,20 \pm 0,59$ & $7,87 \pm 0,74$ & $<0,0001$ \\
Гликемия натощак, ммоль/л & $7,14 \pm 1,46$ & $8,31 \pm 1,86$ & $<0,0001$ \\
Индекс НОМА-В & $53,22 \pm 36,95$ & $39,67 \pm 24,74$ & 0,033 \\
Концентрация альбумина в плазме, г/л & $46,18 \pm 2,51$ & $45,23 \pm 2,30$ & 0,024 \\
ЛПВП, ммоль/л & $1,36 \pm 0,28$ & $1,26 \pm 0,26$ & 0,076 \\
ТГ/ЛПВП & $0,87 \pm 0,70$ & $1,48 \pm 0,95$ & 0,079 \\
Концентрация АЛТ, Ед/л & $25,92 \pm 15,55$ & $28,10 \pm 13,09$ & 0,062 \\
\hline
\end{tabular}

Построение многофакторной модели линейной регрессии

Поскольку российская и корейская субпопуляции не отличались по НbА 1 в начале терапии $(p=0,696)$ и имели сопоставимое снижение $\mathrm{HbA}_{1 c}$ через 24 нед $(\mathrm{p}=0,690)$, представляется возможным объединить субпопуляции для последующего анализа.

В многофакторную модель вошли данные 132 пациентов: у одного пациента отсутствовали данные о НОМА-В индексе, у одного - о соотношении триглицеридов и ЛПВП, у 6 пациентов не было значения $\mathrm{HbA}_{1 с}$ через 24 нед терапии.

В табл. 3 приведены характеристики многофакторной модели линейной регрессии. Снижение $\mathrm{HbA}_{1 с}$ через 24 нед было тем более выражено, чем выше был исходный $\mathrm{HbA}_{1 c}(p<0,0001)$ и индекс НОМА-B $(p=0,0042)$. Лог-трансформация индекса НОМА-В не влияет на направление связи: чем выше соотношение инсулина и гликемии натощак, тем более эффективным с точки зрения гипогликемического эффекта будет назначение эвоглиптина. Повышение соотношения концентрации триглицеридов и ЛПВП в крови, напротив, было ассоциировано с меньшей гипогликемической эффективностью эвоглиптина ( $p=0,0057)$. Прием статинов также снижал эффективность эвоглиптина $(p=0,044)$, при этом взаимодействие факторов приема статинов и соотношения триглицеридов и ЛПВП не является статистически значимым ( $p=0,88)$. Более высокая концентрация фосфора в крови также была ассоциирована с меньшей эффективностью эвоглиптина ( $p=0,014)$. Факторы, включенные в модель, определяют 30,3\% вариабельности.

Учет конфаундинг-эффекта

Характеристики однофакторных моделей линейной регрессии для параметров, включенных в многофактор- ную модель, приведены в табл. 3 для оценки влияния конфаундеров. Линейные коэффициенты для факторов исходного уровня $\mathrm{HbA}_{1 с}$ и концентрации фосфора менялись в пределах допустимых значений, что позволяет говорить об отсутствии эффекта конфаундинга для этих ковариат.

Конфаундерами для индекса НОМА-В выступают составляющие самого индекса, а именно - концентрации глюкозы и инсулина натощак на старте терапии. Замена индекса НОМА-В на эти два фактора в многофакторной модели линейной регрессии показывает разнонаправленный вклад в эффект исходного значения гликемии натощак $(B=-0,089 ; p=0,02)$ и концентрации инсулина $(B=0,016 ; p=0,06)$, что логично следует из формулы расчета индекса НОМА-В. При этом раздельное рассмотрение компонентов индекса НОМА-В в рамках многофакторной модели линейной регрессии не влияет на процент вариабельности, определяемый моделью.

Конфаундеры для ковариат «прием статинов» и «триглицериды/ЛПВП» не были выявлены по существующей базе данных. Тем не менее следует принять во внимание, что полученная модель предсказывает снижение $\mathrm{HbA}_{1 с}$ через 24 нед в среднем на 0,51\% меньше, чем его фактическое снижение (95\% ДИ: 0,39-0,62\%), что отчасти может быть связано с линейными коэффициентами вышеупомянутых ковариат.

\section{Характеристики пациентов, достигших вторичной} конечной точки

Пациенты, достигшие целевых значений $\mathrm{HbA}_{1 c} \leq 7,0 \%$, имели меньший уровень $\mathrm{HbA}_{1 c}(\mathrm{p}<0,0001)$ и гликемии натощак $(p<0,0001)$ на старте терапии. Индекс НОМА-В у этих пациентов был выше, чем у тех, кто не достиг цели $\leq 7,0 \%(p=0,033)$, и концентрация альбумина в плазме крови была выше ( $p=0,024)$. Также отмечалась тенденция 
к более высокому содержанию ЛПВП $(p=0,076)$ в плазме крови, а также к более низкому соотношению триглицериды/ЛПВП ( $p=0,079)$ и концентрации АЛТ $(p=0,062)$. Для данных показателей размер выборки был слишком мал для достижения достаточной статистической мощности. Средние значения ленных показателей приведены в табл. 4. Все показатели оценены на старте терапии.

\section{ОБСУЖДЕНИЕ}

Резюме основного результата исследования

Факторами, ассоциированными с более выраженным гипогликемическим эффектом эвоглиптина, являются исходный $\mathrm{HbA}_{1 c^{\prime}}$ индекс НОМА-В, МИ и концентрация фосфора в крови пациентов, которым была инициирована терапия эвоглиптином.

Параметры гликемического контроля: $\mathrm{HbA}_{1 c^{\prime}}$

HOMA-B индекс, HOMA-IR индекс, QUICKI индекс,

метаболический индекс

По результатам РКИ, база данных которого была основным источником данных для настоящего исследования, назначение эвоглиптина в дозе 5 мг позволяло достичь среднего снижения уровня $\mathrm{HbA}_{1 с}$ на 0,60\% (95\% ДИ: 0,48-0,71\%), что объясняет меньшую вероятность достижения целевого значения $\mathrm{HbA}_{1 c} \leq 7,0 \%$ у пациентов с более высокими исходными значениями данного показателя. В то же время выраженность гипогликемического эффекта была прямо пропорциональна исходному $\mathrm{HbA}_{1 c}$ - так же, как и для других лекарственных препаратов класса иДПП-4 [5, 6].

В настоящем исследовании была получена положительная корреляция индекса НОМА-В как с эффективностью терапии, так и с вероятностью достижения целевых значений $\mathrm{HbA}_{1 c}$. Данный эффект для обеих конечных точек достигался прежде всего за счет более низкой концентрации глюкозы плазмы натощак. При этом, в отличие от результатов, полученных для ситаглиптина [17], индекс инсулинорезистентности HOMA-IR в настоящем исследовании не был ассоциирован с эффективностью терапии эвоглиптином.

Соотношение концентрации триглицеридов и лПВП в плазме крови (МИ) имело отрицательную корреляцию с гипогликемической эффективностью эвоглиптина и было ниже в группе пациентов, достигших целевых значений $\mathrm{HbA}_{1 c}$. В данном случае вклад вносят оба параметра, используемых для расчета МИ: и более низкая концентрация ТГ, и более высокая концентрация ЛПВП ассоциированы с лучшим ответом на терапию. Основным преимуществом данного индекса перед НОМА-В является доступность определения его компонент, тогда как измерение концентрации инсулина в плазме не всегда представляется возможным, и с учетом ранее доказанной удовлетворительной точности МИ $[25,26]$ представляется целесообразным рассматривать его как самостоятельный предиктор эффективности терапии эвоглиптином.

В настоящем исследовании была также выявлена положительная корреляция между снижением $\mathrm{HbA}_{1 с}$ через 24 нед терапии и соотношением индексов QUICKI через 24 нед и на старте терапии $(B=1,77 ; p=0,018)$. Данный факт не может быть использован для прогноза эффективности, но служит еще одним подтверждением полученных результатов. Описанное соотношение индексов QUICKI математически представляет собой соотношение двух логарифмов:

(log глюкоза * инсулин) [на старте терапии])

(log глюкоза * инсулин) [через 24 недели]),

что, в свою очередь, соответствует соотношению логарифмов индексов HOMA-IR на старте терапии и через 24 нед. Таким образом, в настоящем исследовании косвенно было получено доказательство прямой корреляции между снижением $\mathrm{HbA}_{1 c}$ и снижением инсулинорезистентности, при этом второе, вероятнее всего, является основой ответа на терапию эвоглиптином, а изменение $\mathrm{HbA}_{1 c}$ - это следствие снижения инсулинорезистентности.

Концентрация фосфора в плазме крови как

прогностический фактор ответа на терапию эвоглиптином

Согласно полученной однофакторной модели линейной регрессии, при концентрации фосфора в плазме крови выше 1,13 ммоль/л снижение $\mathrm{HbA}_{1 c}$ через 24 нед будет меньше 0,6\% (меньше среднего значения для всех пациентов, принимавших эвоглиптин). Концентрация фосфора в плазме крови, по-видимому, является самостоятельным прогностическим фактором, не подверженным конфаундинг-эффекту, как в однофакторной, так и в многофакторной модели линейной регрессии.

Ранее было показано, что у здоровых добровольцев уровень гликемии был прямо пропорционален концентрации фосфора в плазме крови $(p=0,042)$, но у пациентов с СД2 данная корреляция не была статистически значимой [27]. Одним из вероятных патофизиологических механизмов, лежащих в основе ассоциации между уровнем гликемии и концентрацией фосфора в плазме крови, в настоящий момент считается хроническое повышение концентрации паратгормона и фактора роста фибробластов-23 (FGF-23), которые регулируют абсорбцию избыточно поступающего фосфора в тонком кишечнике [28]. Повышенный уровень FGF-23 ассоциирован с инсулинорезистентностью [29], что, в свою очередь, может объяснять прямую корреляцию между концентрацией фосфора в плазме крови и уровнем гликемии [30].

Таким образом, повышение концентрации фосфора в плазме крови ассоциировано с повышением инсулинорезистентности (опосредованно через FGF-23), а гипогликемическая эффективность эвоглиптина при нарастании инсулинорезистентности снижается, что объясняет выявленную в настоящем исследовании корреляцию. На данный момент неизвестно, актуальна ли эта ассоциация для других лекарственных препаратов класса иДПП-4 (на основании данных о патофизиологическом механизме), или она является значимой только для эвоглиптина, что требует дальнейшего изучения.

Роль сопутствующей терапии у пациентов

В многофакторной модели линейной регрессии в настоящей модели было получено статистически значимое негативное влияние приема статинов на гипогликемическую эффективность эвоглиптина $(p=0,044)$. Данный факт, вероятно, следует рассматривать в комплексе 
с показателями МИ (триглицериды/ЛПВП) и с индексом инсулинорезистентности HOMA-IR, поскольку ранее было показано, что прием статинов приводит к снижению чувствительности к инсулину и его секреции [31]. Casula и соавт. опубликовали результаты мета-анализа 20 наблюдательных исследований, которые показали 44\% повышение вероятности развития СД при приеме статинов [32], что подтвердило ранее полученные данные [33]. Гипергликемический эффект приема статинов имел место и у пациентов с уже диагностированным СД2: пациенты, принимавшие статины, имели на 0,12\% больший показатель $\mathrm{HbA}_{1 c^{\prime}}$ чем те, кто статины не принимал $(p=0,003)$, при этом авторы отмечают, что гипогликемическая терапия могла быть не сбалансирована между группами, а полученный статистический эффект не является клинически значимым и потому не должен быть препятствием к назначению статинов с целью снижения риска сердечно-сосудистых исходов [34]. В настоящем исследовании вклад приема статинов в прогнозируемый эффект эвоглиптина также следует принять во внимание, однако он не должен быть решающим при выборе терапии.

В настоящем исследовании также было получено на 0,22\% большее снижение $\mathrm{HbA}_{1 с}$ через 12 нед терапии при назначении эвоглиптина у пациентов, принимавших ингибиторы ангиотензинпревращающего фермента (иАПФ) и блокаторы рецепторов ангиотензина II (БРА), по сравнению с теми, кто не получал данные препараты $(p=0,042)$. Данный эффект был достигнут прежде всего за счет приема БРА $(p=0,031)$, но не за счет приема иАПФ $(p=0,99)$. Данный факт не был вынесен в раздел результатов исследования, поскольку различия в степени снижения $\mathrm{HbA}_{1 с}$ не сохранились через 24 нед терапии $(p=0,41)$. Тем не менее эффект, полученный в настоящем исследовании, не является случайным: ранее было показано, что прием иАПФ и БРА снижает риск развития СД в общей популяции на 20 и 27\% соответственно [35], а при уже диагностированном СД назначение БРА ассоциировано со снижением выраженности оксидативного стресса и системного воспаления, ведущих к повышению инсулинорезистентности [36]. Таким образом, положительная корреляция гипогликемического эффекта инкретинов и приема препаратов, влияющих на РААС, вероятно, не ограничивается эвоглиптином, а характерна для всего класса как иДПП-4, так и аГПП-1, что требует дальнейшего изучения.

\section{Ограничения исследования}

Основным ограничением исследования является его ретроспективный характер и возможная неоднородность исходных данных, включенных в анализ. Тем не менее использование многофакторной модели линейной регрессии позволяет преодолеть возможный конфаундинг эффект и модификацию эффекта, вызванные потенциальной неоднородностью базовых характеристик.

Вторым ограничением является невозможность установления причинно-следственной связи только на основании результатов статистического анализа, что является ограничением выбранного метода статистической обработки данных. Это ограничение частично преодолено в разделе Обсуждения настоящей статьи.

\section{ЗАКЛЮЧЕНИЕ}

Пациент, который получит максимальную гликемическую выгоду от применения эвоглиптина, - это пациент с умеренной гипергликемией, с более высоким индексом НОМА-В, с меньшим МИ и с низко-нормальной концентрацией фосфора в плазме крови.

В настоящем исследовании не было получено корреляции между снижением $\mathrm{HbA}_{1 с}$ через 24 нед и ИМТ, длительностью СД и концентрацией С-пептида в плазме крови, в отличие от данных, полученных для других иДПП-4 [6,7]. Количество пациентов с ишемической болезнью сердца в настоящей выборке было недостаточным для проведения статистического анализа.

Представленные данные позволяют усилить полученные ранее данные о предикторной ценности исходного уровня $\mathrm{HbA}_{1 с}$ и выраженности инсулинорезистентности в ответ на терапию иДПП-4. В то же время они поднимают вопрос о наличии различий в предикторах ответа на отдельных представителей данной группы, что требует тщательного анализа предикторов для каждого представителя класса препаратов.

\section{ДОПОЛНИТЕЛЬНАЯ ИНФОРМАЦИЯ}

Источник финансирования. Исследование выполнено при финансовой поддержке гранта Российского научного фонда (проект № 17-75-30052) и софинансирования, предоставленного научному проекту № 17-75-30052 ООО «Герофарм».

Конфликт интересов. Для математического анализа, выполненного для идентификации предикторов, использовалась база данных рандомизированного клинического исследования ЭВОКОМБИ, спонсором которого являлось ООО «Герофарм». Между тем статья не содержит промоционных материалов и основана на беспристрастном анализе данных.

Участие авторов. Мосикян А.А. - статистический анализ предоставленных данных, написание первичного текста рукописи; Бабенко А.Ю. - координирующий исследователь двойного слепого рандомизированного клинического испытания по сравнению эффективности и безопасности эвоглиптина 5 мг и ситаглиптина 100 мг, главный исследователь в ФГБУ «НМИЦ им. В.А. Алмазова», корректура и рецензирование первичного текста рукописи; Севастьянова Ю.А. - статистический анализ предоставленных данных; Драй Р.В. - обеспечение проведения двойного слепого рандомизированного клинического испытания по сравнению эффективности и безопасности эвоглиптина 5 мг и ситаглиптина 100 мг, подготовка протокола исследования, контроль его проведения в центрах, корректура и рецензирование первичного текста рукописи; Шляхто Е.В. - корректура и рецензирование всех версий рукописи. Все авторы внесли существенный вклад в проведение исследования и подготовку статьи, прочли и одобрили финальную версию перед публикацией.

Благодарности. Авторы выражают благодарность исследователям и исследовательским центрам, принимавшим участие в исследовании эвОКОМБИ.

На территории Российской Федерачии:

1. ГБУЗ города Москвы «Эндокринологический диспансер Департамента здравоохранения города Москвы». Главный исследователь - Анциферов Михаил Борисович, д.м.н., профессор; главный врач ГБУз «Эндокринологический диспансер ДЗМ»; профессор ФГАОУ ВО Первый МгмУ им. И.М. Сеченова Минздрава России.

2. СПб ГБУз «Городская больница Святой преподобномученицы Елизаветы» (Елизаветинская больница). Главный исследователь - 
Ворохобина Наталья Владимировна, д.м.н., профессор; научный консультант эндокринологического отделения СПб ГУз «Елизаветинская больница»; заведующая кафедрой эндокринологии, профессор ФГБОУ ВО «Северо-Западный государственный медицинский университет им. И.И. Мечникова» Минздрава России.

3. ФГБУ «Национальный медицинский исследовательский центр им. В.А. Алмазова» Министерства здравоохранения Российской Федерации. Главный исследователь - Бабенко Алина Юрьевна, д.м.н., доцент кафедры внутренних болезней института послевузовского образования НМИЦ, заведующая НИЛ диабетологии Института эндокринологии НМИЦ, заместитель директора Института эндокринологии по учебной работе, врач-эндокринолог высшей категории.

4. ФГБУ «Научный медицинский исследовательский центр эндокринологии» Минздрава России. Главный исследователь - Майоров Александр Юрьевич, д.м.н., заведующий отделом прогнозирования и инноваций диабета ФГБУ «НМИЦ эндокринологии» Минздрава России.

5. Филиал негосударственного частного учреждения здравоохранения «Научный клинический центр» Открытого акционерного общества «Российские железные дороги». Главный исследователь - Войчик Эмма Анатольевна, к.м.Н., руководитель эндокринологического центра НУЗ «НКЦ ОАО «РЖД»».

6. ФГАОУ ВО «Первый Московский государственный медицинский университет им. И.М. Сеченова» Минздрава России. Главный исследователь - Петунина Нина Александровна, д.м.н., профессор, заведующая кафедрой эндокринологии ФГАОУ ВО «Первый МГМУ им. И.М. Сеченова» Минздрава России.

Ha территории Южной Кореи:

7. Больница Кангбук Самсунг (Kangbuk Samsung Hospital). Главный исследователь - Сунг-Ву Пак, профессор отделения эндокринологии больницы Кангбук Самсунг (Prof. Sung-Woo Park, Department of Endocrinology, Kangbuk Samsung Hospital).

8. Больница Пресвятого Сердца университета Халлим Кандонга (Hallym University Gangdong Sacred Heart Hospital). Главный исследователь - Ду Ман Ким, профессор отделения эндокринологии и метаболизма больницы Святого Сердца Кандонгу университета Халлим (Prof. Doo Man Kim, Department of Endocrinology and Metabolism, Hallym University Gangdong Sacred Heart Hospital).

9. Медицинский центр Гиль Университета Гачон (Gachon University Gil Medical Center). Главный исследователь - Ки Ёнг Ли, доцент отделения эндокринологии и метаболизма Медицинского центра Гиль Университета Гачон (Assistant Prof. Ki Young Lee, Department of Endocrinology and Metabolism, Gachon University Gil Medical Center).

10. Католический университет Кореи, больница Святой Марии в Сеуле (The Catholic University of Korea Seoul St. Mary's Hospital). Главный исследователь - Кун Хо Юн, профессор отделения эндокринологии и метаболизма больницы Св. Марии Католического Университета Сеула (Prof. Kun Ho Yoon, Catholic University Seoul St. Mary's Hospital).

11. Католический университет Кореи, больница Святой Марии в Пучхоне (Catholic University of Korea Bucheon St. Mary's Hospital). Главный исследователь - Сунг Рэ Ким, доцент отделения эндокринологии и метаболизма больницы Св. Марии Католического университета Бучон (Associate Prof. Sung Rae Kim, Catholic University Bucheon St. Mary's Hospital).

12. Католический университет Кореи, больница Святой Марии в Йеуидо (The Catholic University of Korea Yeouido St. Mary's Hospital). Главный исследователь - Ки Хо Сонг, профессор отделения эндокринологии и метаболизма (Prof. Ki Ho Song, Department of Endocrinology and Metabolism, Catholic University Yeouido St. Mary's Hospital).
13. Больница университета Кёнхи в Кандонге (Kyung Hee University Hospital at Gangdong). Главный исследователь - Кён Чын Ан, профессор отделения эндокринологии больницы университета Кёнхи в Кандонгу (Prof. Kyu Jeung Ahn, Department of Endocrinology Gangdong Kyung Hee University Hospital).

14. Больница Кёнбукского национального университета (Kyungpook National University Hospital). Главный исследователь - Ин Кё Ли, профессор отделения эндокринологии больницы Кенбукского национального университета (Prof. In Kyu Lee, Kyungpook National University Hospital).

15. Больница Куро Корейского университета (Korea University Guro Hospital). Главный исследователь - Кунг Мук Чой, профессор отделения эндокринологии и метаболизма больницы Куро Корейского университета (Prof. Kyung Mook Choi, Department of Endocrinology and Metabolism, Korea University Guro Hospital).

16. Больница Мёнджи (Myongji Hospital). Главный исследователь - Джэ Хёк Ли, профессор отделения эндокринологии больницы Мёнджи (Prof. Jae Hyuck Lee, Department of Endocrinology, Myong Ji Hospital.

17. Медицинский центр Самсунг (Samsung Medical Center). Главный исследователь - Мун Кё Ли, профессор отделения эндокринологии и метаболизма Медицинского центра Самсунг (Prof. Moon Kyu Lee, Department of Endocrinology, Samsung Medical Hospital).

18. Больница Сеульского национального университета (Seoul National University Hospital). Главный исследователь - Кён Су Пак, профессор отделения эндокринологии, метаболизма и внутренних болезней больницы Сеульского университета (Prof. Kyong Soo Park, Department of Endocrinology, Metabolism and Internal Disease, Seoul National University Hospital).

19. Медицинский центр Асан (Asan Medical Center). Главный исследователь - Чун Йол Пак, профессор отделения эндокринологии и метаболизма Медицинского центра Асан (Prof. Joong Yeol Park, Department of Endocrinology and Metabolism, Asan Medical Center).

20. Больница университета Сун Чон Хён, Пучхон (Soon Chun Hyang University Hospital Bucheon). Главный исследователь - Чи О Мок, профессор отделения эндокринологии Сун Чун Хён больницы университета Пучхон (Prof. Ji Oh Mok, Department of Endocrinology, Bucheon Soonchunhyang University Bucheon Hospital).

21. Больница Северанс, учреждение здравоохранения при университете Йонсей (Severance Hospital, Yonsei University Health System). Главный исследователь - Бон Су Чха, профессор отделения эндокринологии, больница Северанс, учреждение здравоохранения при университете Йонсей (Prof. Bong Soo Cha, Department of Endocrinology, Yonsei University Severance Hospital).

22. Христианская больница Вонджу Северанс (Wonju Severance Christian Hospital). Главный исследователь - Профессор Чхун Хи Чун, Отделение эндокринологии, Христианская больница Вонджу Северанс (Prof. Choon Hee Chung, Department of Endocrinology, Wonju Severance Christian Hospital).

23. Больница общего типа Ыльчи (Eulji General Hospital). Главный исследователь - Кён А Хан, профессор отделения эндокринологии, больница общего типа Ыльчи (Prof. Kyung Ah Han, Department of Endocrinology, Eulji General Hospital).

24. Университет Индже, больница Пусан Пэк (Inje University Busan Paik Hospital). Главный исследователь - Чон Хён Пак, профессор отделения эндокринологии и лечения внутренних болезней, университет Индже больница Пусан Бек (Prof. Jeong Hyun Park, Department of Endocrinology, Internal Medicine, Inje University Busan Paik Hospital).

25. Больница университета Инха (Inha University Hospital). Главный исследователь - Сон Бин Хон, доцент отделения эндокринологии и метаболизма, больница университета Инха (Associate Prof. Seong Bin Hong, Department of Endocrinology and Metabolism, Inha University Hospital). 
26. Больница Национального университета Чоннам (Chonnam National University Hospital). Главный исследователь - Мин Ян Чун, профессор отделения эндокринологии и метаболизма, больница Национального университета Чоннам (Prof. Min Young Chung, Department of Endocrinology and Metabolism, Chonnam National University Hospital).

27. Больница национального университета Чеджудо (Jејu National Universiy Hospital). Главный исследователь - Гван Пё Ко, доцент отделения эндокринологии и метаболизма, больница национального университета Чеджу (Prof. Gwan Pyo Koh, Department of Endocrinology and Metabolism, Jeju National University Hospital).

28. Больница Пресвятого Сердца университета Халлим (Hallym University Sacred Heart Hospital). Главный исследователь - Чон Гу Кан, доцент отделения эндокринологии и метаболизма, больница Пресвятого Сердца университета Халлим (Associate Prof. Jun Goo Kang, Department of Endocrinology and Metabolism, Hallym University Sacred Heart Hospital).

29. Больница Кури университета Ханъян (Hanyang University Guri Hospital). Главный исследователь - Чан Пом Ли, профессор отделения эндокринологии и метаболизма, больница Кури университета
Ханъян (Prof. Chang Beom Lee, Department of Endocrinology and Metabolism, Hanyang University Guri Hospital).

30. Больница Мокдон женского университета Ихва (Ewha Womans University Mokdong Hospital). Главный исследователь - Ён А Сун, профессор отделения эндокринологии, больница Мокдон, женский университет Ихва (Prof. Yeon Ah Sung, Division of Endocrinology, Ewha Womans University Mokdong Hospita).

31. Университетская больница Конянг (Konyang University Hosptial). Главный исследователь - Кён Ён Пак, профессор отделения эндокринологии, университетская больница Конянг (Prof. Keun Yong Park, Department of Endocrinology, Konyang University Hospital).

32. Больница Пундан Сеульского национального университета (Seoul National University Bundang Hospital). Главный исследователь - Су Лим, доцент отделения эндокринологии, больница Пундан, Сеульский национальный университет (Associate Prof. Su Lim, Department of Endocrinology, Seoul National University Bundang Hospital).

33. Медицинский центр Йоннамского университета (Yeungnam University Medical Center). Главный исследователь - Хён Ву Ли, профессор отделения эндокринологии и метаболизма, Медицинский центр Йоннамского университета (Prof. Hyung Woo Lee, Department of Endocrinology and Metabolism, Yongnam University Hospital).

\section{СПИСОК ЛИТЕРАТУРЫ | REFERENCES}

1. Шляхто Е.В., Конради А.О. Основные направления и перспективы трансляционных исследований в кардиологии // Вестник Росздравнадзора. - 2015. - №5. - C. 33-37. [Shlyakhto EV, Konradi AO. Key areas and prospects for translational research in cardiology. Vestnik Roszdravnadzora. 2015;(5):33-37 (In Russ.)]

2. Mancinelli L, Cronin M, Sadée W. Pharmacogenomics: The promise of personalized medicine. AAPS PharmSci. 2000;2(1):29-41. doi: 10.1208/ps020104

3. Committee on A Framework for Developing a New Taxonomy of Disease, Board on Life Sciences, Division on Earth and Life Studies. Toward Precision Medicine: Building a Knowledge Network for Biomedical Research and a New Taxonomy of Disease. Washington: National Academy of Sciences; 2011.

4. Tan X, Hu J. Evogliptin: a new dipeptidyl peptidase inhibitor for the treatment of type 2 diabetes. Expert Opin Pharmacother. 2016;17(9):1285-1293. doi: 10.1080/14656566.2016.1183645

5. Esposito K, Chiodini P, Maiorino MI, et al. A nomogram to estimate the $\mathrm{HbA} 1 \mathrm{C}$ response to different DPP-4 inhibitors in type 2 diabetes: a systematic review and meta-analysis of 98 trials with 24163 patients. BMJ Open. 2015;5(2):e005892. doi: 10.1136/bmjopen-2014-005892

6. Yagi S, Aihara K, Akaike M, et al. Predictive Factors for Efficacy of Dipeptidyl Peptidase-4 Inhibitors in Patients with Type 2 Diabetes Mellitus. Diabetes Metab J. 2015;39(4):342-347. doi: 10.4093/ dmj.2015.39.4.342

7. Mamza J, Mehta R, Donnelly R, Idris I. Determinants of Glycemic Response to Add-On Therapy with a Dipeptidyl Peptidase-4 Inhibitor: A Retrospective Cohort Study Using a United Kingdom Primary Care Database. Diabetes Technol Ther. 2016;18(2):85-92. doi: 10.1089/dia.2015.0052

8. Monami M, Cremasco F, Lamanna C, et al. Predictors of response to dipeptidyl peptidase-4 inhibitors: evidence from randomized clinical trials. Diabetes Metab Res Rev. 2011;27(4):362-372. doi: 10.1002/dmrr.1184

9. Kim YG, Hahn S, Oh TJ, et al. Differences in the glucose-lowering efficacy of dipeptidyl peptidase-4 inhibitors between Asians and non-Asians: a systematic review and meta-analysis. Diabetologia. 2013;56(4):696-708. doi: 10.1007/s00125-012-2827-3

10. Kahn SE, Hull RL, Utzschneider KM. Mechanisms linking obesity to insulin resistance and type 2 diabetes. Nature. 2006;444(7121):840846. doi: 10.1038/nature05482

11. Jensen CC, Cnop M, Hull RL, et al. -Cell Function Is a Major Contributor to Oral Glucose Tolerance in High-Risk Relatives of Four Ethnic Groups in the U.S. Diabetes. 2002;51(7):2170-2178. doi: $10.2337 /$ diabetes.51.7.2170

12. Jamaluddin JL, Huri HZ, Vethakkan SR. Clinical and genetic predictors of dipeptidyl peptidase-4 inhibitor treatment response in
Type 2 diabetes mellitus. Pharmacogenomics. 2016;17(8):867-881. doi: $10.2217 /$ pgs-2016-0010

13. Zhou K, Pedersen HK, Dawed AY, Pearson ER. Pharmacogenomics in diabetes mellitus: insights into drug action and drug discovery. Nat Rev Endocrinol. 2016;12(6):337-346. doi: 10.1038/nrendo.2016.51

14. Tkac I, Gotthardova I. Pharmacogenetic aspects of the treatment of Type 2 diabetes with the incretin effect enhancers. Pharmacogenomics. 2016;17(7):795-804. doi: 10.2217/pgs-2016-0011

15. Zimdahl H, Ittrich C, Graefe-Mody U, et al. Influence of TCF7L2 gene variants on the therapeutic response to the dipeptidylpeptidase-4 inhibitor linagliptin. Diabetologia. 2014;57(9):1869-1875. doi: 10.1007/s00125-014-3276-y

16. Oh TJ, Jung HS, Bae JH, et al. Clinical characteristics of the responders to dipeptidyl peptidase-4 inhibitors in Korean subjects with type 2 diabetes. J Korean Med Sci. 2013;28(6):881-887. doi: 10.3346/jkms.2013.28.6.881

17. Kim HM, Lim JS, Lee BW, et al. Optimal candidates for the switch from glimepiride to sitagliptin to reduce hypoglycemia in patients with type 2 diabetes mellitus. Endocrinol Metab (Seoul). 2015;30(1):84-91. doi: 10.3803/EnM.2015.30.1.84

18. Del Prato S, Patel S, Crowe S, von Eynatten M. Efficacy and safety of linagliptin according to patient baseline characteristics: A pooled analysis of three phase 3 trials. Nutr Metab Cardiovasc Dis. 2016;26(10):886-892. doi: 10.1016/j.numecd.2016.06.015

19. Sell $H$, Bluher $M$, Kloting $N$, et al. Adipose dipeptidyl peptidase-4 and obesity: correlation with insulin resistance and depot-specific release from adipose tissue in vivo and in vitro. Diabetes Care. 2013;36(12):4083-4090. doi: 10.2337/dc13-0496

20. Maeda H, Kubota A, Tanaka Y, et al. The safety, efficacy and predictors for $\mathrm{HbA} 1 \mathrm{c}$ reduction of sitagliptin in the treatment of Japanese type 2 diabetes. Diabetes Res Clin Pract. 2012;95(1):e20-22. doi: 10.1016/j.diabres.2011.10.011

21. Nomiyama T, Akehi $\mathrm{Y}$, Takenoshita $\mathrm{H}$, et al. Contributing factors related to efficacy of the dipeptidyl peptidase-4 inhibitor sitagliptin in Japanese patients with type 2 diabetes. Diabetes Res Clin Pract 2012;95(2):e27-28. doi: 10.1016/j.diabres.2011.08.016

22. Pratley RE, McCall T, Fleck PR, et al. Alogliptin use in elderly people: a pooled analysis from phase 2 and 3 studies. J Am Geriatr Soc. 2009;57(11):2011-2019. doi: 10.1111/j.1532-5415.2009.02484.x

23. Katz A, Nambi SS, Mather K, et al. Quantitative insulin sensitivity check index: a simple, accurate method for assessing insulin sensitivity in humans. J Clin Endocrinol Metab. 2000;85(7):2402-2410. doi: 10.1210/jcem.85.7.6661

24. Chen H, Sullivan G, Quon MJ. Assessing the Predictive ACcuracy of QUICKI as a Surrogate Index for Insulin Sensitivity 
Using a Calibration Model. Diabetes. 2005:54(7):1914-1925. doi: 10.2337/diabetes.54.7.1914

25. Kim-Dorner SJ, Deuster PA, Zeno SA, et al. Should triglycerides and the triglycerides to high-density lipoprotein cholesterol ratio be used as surrogates for insulin resistance? Metabolism. 2010;59(2):299-304. doi: 10.1016/j.metabol.2009.07.027

26. Roytberg GE, Dorosh JV, Sharkhun OO, et al. New Metabolic Index Use Potentialities in Evaluation of Insulin Resistance in Clinical Practice. Rational Pharmacotherapy in Cardiology. 2014;10(3):264-274. doi: 10.20996/1819-6446-2014-10-3-264-274

27. Fang L, Li X. Level of serum phosphorus and adult type 2 diabetes mellitus. Zhong Nan Da Xue Xue Bao Yi Xue Ban. 2016;41(5):502-506. doi: 10.11817/j.issn.1672-7347.2016.05.009

28. Martin A, David V, Quarles LD. Regulation and function of the FGF23/klotho endocrine pathways. Physiol Rev. 2012;92(1):131-155. doi: 10.1152/physrev.00002.2011

29. Mirza MA, Alsio J, Hammarstedt A, et al. Circulating fibroblast growth factor-23 is associated with fat mass and dyslipidemia in two independent cohorts of elderly individuals. Arterioscler Thromb Vasc Biol. 2011;31(1):219-227. doi: 10.1161/ATVBAHA.110.214619

30. Mancini FR, Affret A, Dow C, et al. High dietary phosphorus intake is associated with an increased risk of type 2 diabetes in the large prospective E3N cohort study. Clin Nutr. 2018:37(5):1625-1630. doi: 10.1016/j.clnu.2017.07.025

31. Cederberg H, Stancakova A, Yaluri N, et al. Increased risk of diabetes with statin treatment is associated with impaired insulin sensitivity and insulin secretion: a 6 year follow-up study of the METSIM cohort. Diabetologia. 2015;58(5):1109-1117. doi: 10.1007/s00125-015-3528-5

32. Casula M, Mozzanica F, Scotti L, et al. Statin use and risk of new-onset diabetes: A meta-analysis of observational studies. Nutr Metab Cardiovasc Dis. 2017;27(5):396-406. doi: 10.1016/j.numecd.2017.03.001

33. Betteridge DJ, Carmena R. The diabetogenic action of statins - mechanisms and clinical implications. Nat Rev Endocrinol. 2016;12(2):99110. doi: 10.1038/nrendo.2015.194

34. Ray KK, Seshasai SRK, Wijesuriya S, et al. Effect of intensive control of glucose on cardiovascular outcomes and death in patients with diabetes mellitus: a meta-analysis of randomised controlled trials. Lancet. 2009;373(9677):1765-1772. doi: 10.1016/s0140-6736(09)60697-8

35. Li Z, Li Y, Liu Y, et al. Comparative risk of new-onset diabetes mellitus for antihypertensive drugs: A network meta-analysis. J Clin Hypertens (Greenwich). 2017;19(12):1348-1356. doi: 10.1111/jch.13108

36. Das UN. Renin-angiotensin-aldosterone system in insulin resis tance and metabolic syndrome. J Trans/ Int Med. 2016;4(2):66-72. doi: 10.1515/jtim-2016-0022

\section{ИНФОРМАЦИЯ ОБ АВТОРАХ [AUTHORS INFO]}

*Бабенко Алина Юрьевна, д.м.н., доцент [Alina Y. Babenko, MD, PhD, associate professor]; адрес: Россия, 197341, Санкт-Петербург, ул. Аккуратова, д. 2 [address: 2, Akkuratova Street, Saint-Petersburg, 197341 Russian Federation]; ORCID: https://orcid.org/0000-0002-0559-697X; eLibrary SPIN: 9388-1077; e-mail: alina_babenko@mail.ru

Мосикян Анна Альбертовна [Anna A. Mosikian]; ORCID: https://orcid.org/0000-0002-2863-270X; eLibrary SPIN: 96056480; e-mail: mosikian.anna@gmail.com

Шляхто Евгений Владимирович, д.м.Н., профессор, академик PAH [Evgenij V. Shlyakhto, MD, PhD, Professor]; ORCID: https://orcid.org/0000-0003-2929-0980 eLibrary SPIN: 6679-7621, e-mail: e.shlyakhto@almazovcentre.ru Драй Роман Васильевич, к.M.н. [Roman V. Drai, MD, PhD]; ORCID: https://orcid.org/0000-0003-4594-6097; eLibrary SPIN: 5271-0404; e-mail: roman.drai@geropharm.com

Севастьянова Юлия Александровна [Yulia A. Sevastyanova]; ORCID: https://orcid.org/0000-0002-7305-1680; eLibrary SPIN: 2867-3711; e-mail: Yuliya.Sevastyanova@geropharm.com

ЦИТИРОВАТЬ:

Мосикян А.А., Бабенко А.Ю., Севастьянова Ю.А., Драй Р.В., Шляхто Е.В. Предикторы эффективности терапии эвоглиптином в российско-корейской популяции пациентов с сахарным диабетом 2 типа // Сахарный диабет. 2018. — T. 21. — №5. - С. 333-343. doi: 10.14341/DM9547

\section{TO CITE THIS ARTICLE:}

Mosikian AA, Babenko AY, Sevastyanova YA, Drai RV, Shlyakhto EV. Effectiveness prediction of evogliptin treatment in type 2 diabetes mellitus in russian-korean population. Diabetes Mellitus. 2018;21(5):333-343. doi: 10.14341/DM9547 